\title{
Article \\ Different Drop Heights in Bilateral Asymmetry and Interjoint Coordination during Repetitive Drop-Jumps
}

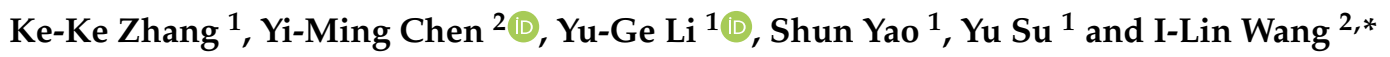 \\ 1 Graduate Institute, Jilin Sport University, Changchun 130022, China; Zhkeke95@st.btbu.edu.cn (K.-K.Z.); \\ liyuge341126@st.btbu.edu.cn (Y.-G.L.); yaoshun@st.btbu.edu.cn (S.Y.); yu.su@st.btbu.edu.cn (Y.S.) \\ 2 College of Physical Education, Hubei Normal University, Huangshi 435002, China; 1021302@ntsu.edu.tw \\ * Correspondence: ilin@gms.ndhu.edu.tw
}

Citation: Zhang, K.-K.; Chen, Y.-M.; Li, Y.-G.; Yao, S.; Su, Y.; Wang, I.-L. Different Drop Heights in Bilateral Asymmetry and Interjoint Coordination during Repetitive

Drop-Jumps. Symmetry 2021, 13, 1590 https: / / doi.org/10.3390/ sym 13091590

Academic Editors:

Jerzy Malachowski,

Adam Ciszkiewicz and

Grzegorz Milewski

Received: 31 July 2021

Accepted: 25 August 2021

Published: 29 August 2021

Publisher's Note: MDPI stays neutral with regard to jurisdictional claims in published maps and institutional affiliations.

\begin{abstract}
The difference of drop heights will affect the biomechanics of lower extremities during dropjump (DJ) landing. Therefore, this study explored the effects of drop heights and training volumes on interjoint coordination and the side-to-side asymmetry of the lower extremities during landing. Twenty males were randomly assigned to perform 200 DJs (DJs200) from 30, 40 and $50 \mathrm{~cm}$ (drop-jump height (DJH) 30, DJH40 and DJH50) platform. One-way ANOVA repeated measure, using MATLAB software, was used to compare the differences of interjoint coordination, side-to-side asymmetry of ground contact time (GCT) and the maximum impact in vertical ground-reaction forces peak (I-vGRFpeak) in the 1st, 50th, 100th, 150th and 200th jumps (DJ1, DJs50, DJs100, DJs150 and DJs200). To examine whether significant differences exist, the least significant difference's (LSD) method was used for post-hoc comparison. The mean absolute relative phase (MARP) and deviation phase (DP) of hip-knee were lower than DJH50 at DJH30 and DJH40, while side-to-side asymmetry of GCT and I-vGRFpeak were greater than DJH30 and DJH40 at DJH50 within DJs200 (all $p<0.05$ ). However, there was no significant difference in MARP and DP of hip-ankle and knee-ankle. Therefore, training at DJH30 may effectively improve jumping performance and reduce musculoskeletal injury risk.
\end{abstract}

Keywords: continuous relative phase; ground contact time; peak ground-reaction force

\section{Introduction}

The drop jump (DJ) is a plyometric exercise who does maximum vertical jump immediately after jumping from different drop heights [1]. Athletes usually use DJ training to enhance muscle strength and jump performance by stretch-shortening cycle (SSC) mechanism $[2,3]$. However, a lower drop height leads to asymmetrical muscle strength during landing [4], while a higher drop height leads to a decrease in power output due to lack of muscle adjustments [5,6]. Appropriate drop height can improve jumping performance and avoid musculoskeletal injury caused by excessive load of lower extremity muscles due to higher drop heights [7-9]. Therefore, inappropriate drop height may reduce jumping performance and cause lower-extremity musculoskeletal injury. In addition, repeated DJ training at different drop heights may cause neuromuscular fatigue, impair jumping performance and lead to injury. Mechanical loads on lower extremity joints during repeated landing may result in stress fractures and anterior cruciate ligament (ACL) injuries, which may limit performance [10,11]. Accordingly, proper drop height and training volume of repetitive DJ training can help improve jumping performance, and inappropriate jump heights may cause lower-extremity musculoskeletal injury.

The movements of the lower extremity are coupled, and disturbance to the system may cause injury [12]. The dynamic system approach is particularly effective in studying the characteristics of motor coordination and stability under the coupling relationship of the lower extremities [13]. The ability of muscles to control joints in dynamic movement such as jump-landing can be described as interjoint coordination, which provides a deeper understanding of the motor control by the central nervous system [14]. Coordination can 
reduce the load of joints during movement by improving dynamic stability, resulting in efficient and accurate functional movement [15]. Past research has found that good coordination in landing allows the load to be evenly distributed between the lower extremities to better absorb the impact of landing $[16,17]$. The lower-extremity instability in interjoint coordination is one of the main factors leading to injury [18]. Muscle fatigue, after repeated jumps, resulted in greater instability of joint coordination, increasing the risk of injury to the lower extremities [19]. However, most of the biomechanics research on DJs have focused on individual joint movements rather than interjoint coordination and the variability of coordination. Therefore, identifying interjoint coordination and variability during the landing phase may be useful for understanding the effects of different drop heights and training volumes on motor-task control, thereby reducing lower-extremity injury.

The side-to-side asymmetry between the lower extremities are useful for understanding neuromuscular control during jump-landing in athletes [20]. In the DJ technique, the athlete usually leaves the platform on one limb and lands on both limbs [4]. The first lower-extremity limb to leave the platform is called the lead limb, and the other the trail limb [4]. The trail limb may not have enough time to catch up with the lead limb, such that side-to-side asymmetry of landing times were increased when landing at a lower drop height, which may affect the neuromuscular load on both limbs and reduce athletic performance [21,22]. The larger side-to-side asymmetry of impact force may indicate reduced jumping ability and power output, as well as increased risk of lower-extremity injury [23]. In addition, such asymmetry values may provide a useful monitoring tool for athletes [24]. Excessive drop heights during DJ landing leads to larger side-to-side asymmetry and can easily cause lower-extremity injury [25]. Therefore, differences of drop height and in training volume impact lower-extremity asymmetry.

In general, drop height and training volume can affect biomechanical differences during landing, and inappropriate heights or training volumes will lead to lower-extremity musculoskeletal injury. Our main hypothesis is that higher drop heights and fatigue would decrease hip-knee-ankle coordination patterns and coordination variability, as well as the kinetic- and temporal-asymmetry increase at ground contact. Therefore, our aim was to study this the patterns of hip-knee-ankle coordination, interjoint coordination variability, and side-to-side asymmetry, by testing different drop heights and training volumes.

\section{Materials and Methods}

\subsection{Subjects}

Through the effect size of 2.23 as in our previous study, a priori power analysis (G*Power version 3.1.9.4; Heinrich Heine University Düsseldorf, Düsseldorf, Germany) proved that according to the traditional $\alpha(0.05)$ and $\beta(0.80)$ values, at least 12 subjects were required to participate in this study [26]. Twenty healthy, male Division III athletes (age $=21.5 \pm 0.9$ years old, height $=174.6 \pm 4.7 \mathrm{~cm}$, body weight $=67.9 \pm 7.9 \mathrm{~kg}$ ) from Jilin Sport University volunteered to participate in the study. The subjects were asked to maintain their normal dietary patterns during the experiment [27]. The subjects did not have any impairments that would hinder their ability to express maximum performance, such as musculoskeletal injuries or neurological disorders of the lower extremities in the preceding year. After explanating the procedure and possible risks, participants gave their written informed consent (form JLSU-IRB2020004) that was approved by the regional ethics committee.

\subsection{Protocol}

Subjects were allowed a short period of time to familiarize themselves with the test procedure and to perform specific warm-up exercises for the major muscle groups of the lower extremity $(10 \mathrm{~min}$ on a treadmill at $8 \mathrm{~km} / \mathrm{h})$ after anthropometric measures were taken. To control for the potential impact of shoes, all subjects were required to wear standard shoes suitable for the subjects' size, provided by the laboratory. Experiments were conducted at the same time in the afternoon. Before the study, the subjects performed 
a standard dynamic warm-up for the major muscle groups of the lower limbs (running on a treadmill at a speed of $8 \mathrm{~km} / \mathrm{h}$ for $10 \mathrm{~min}$ ). The subjects were asked to avoid training and to maintain a normal diet $24 \mathrm{~h}$ prior to testing sessions. In this study, all subjects performed three random DJ task (200 DJs for each task) over three days from 30, 40, and $50 \mathrm{~cm}$ drop heights (DJH30, DJH40, and DJH50), similar to the height used in previous studies [7], with four-day breaks between each drop-height experiment. Subjects were required to leave the raised platform on one limb, land on both limbs at the same time, and then make a maximum vertical jump immediately after landing in the shortest time of contact with the ground $[4,28]$. Subjects need to land in their natural landing style with both feet touching the ground as symmetrically as possible [27]. To ensure that their hands were on their waists and their feet on two force plates during the test, all subjects were asked to practice the jump five times before data collection. The data for DJ1, DJs50, DJs100, DJs150, and DJs200 were recorded, and a $10 \mathrm{~s}$ interval was maintained between each jump performed.

\subsection{Data Collection}

The tests were conducted using 10 cameras (BTS DX400, BTS Bioengineering, Milano, Italy) at a $200 \mathrm{~Hz}$ sampling rate to record kinematic data, and 2 force platforms $(40 \times 60 \mathrm{~cm})$ sampling at $400 \mathrm{~Hz}$ (BTS P6000, BTS Bioengineering, Milano, Italy) to measure groundreaction forces. A modified Helen Hayes marker set was used to identify a 7-segment rigid-link model of the lower extremity, with 21 reflective markers (19 $\mathrm{mm}$ in diameter) attached to the leg and pelvis [29].

\subsection{Data Analysis}

The ground-reaction force (GRF) data was smoothed by a fourth-order low-pass Butterworth digital filter with a cut-off frequency of $50 \mathrm{~Hz}$. The landing phase was defined as the time interval from the foot contacting the ground (t1) to the knee joint reaching its maximum flexion angle (t2), as analyzed for the dynamic data. The vertical GRF (vGRF) threshold of 10-N was evaluated to determine the moment of foot contact (t1) and the maximum vGRF was defined as I-vGRFpeak during the landing phase. The I-vGRFpeak was normalized to body weight (BW). The I-vGRFpeak and interval between $\mathrm{t} 1$ and $\mathrm{t} 2$ during the landing phase were used to calculate side-to-side asymmetry. Continuous relative phase (CRP), as a measurement, was used to describe the patterns of interjoint coordination and information about the variability of coordination patterns [30], as the variability of coordination can well reflect the adaptability, the degree of sensory feedback, and degree of load-sharing of the lower extremity joints during jump-landing [31]. A value of zero indicates that both joints are moving fully in-phase, and a value of 180 represents a their movement in full antiphase [32]. The mean absolute relative phase (MARP) was used to extract a value describing interjoint coordination (i.e., in-phase/antiphase coupling relationships) for the hip-knee, hip-ankle, and knee-ankle segments. Deviation phase (DP) was calculated by determining the absolute difference in the phase angles of the two adjacent joints over the entire motion cycle. DP represents the standard deviation of each point on the overall curve, and as the average of the standard deviation of the entire profile during the landing phase, to determine interjoint coordination variability during DJ. The phase angle (PA) between joints was calculated to evaluate interjoint coordination during landing. For each joint, the phase diagram of the normalized angular velocity $(\omega)$ relative to the corresponding normalized angle $(\theta)$ is established according to Formula (1), and then the normalized joint angle was calculated according to Formula (2):

$$
\begin{gathered}
\omega_{\mathrm{j}} \text { norm }=\omega / \max \left[\max \left(\omega_{\mathrm{j}}\right), \max \left(-\omega_{\mathrm{j}}\right)\right] \\
\theta_{\mathrm{j}} \operatorname{norm}=2 *\left[\theta_{\mathrm{j}}-\min \left(\theta_{\mathrm{j}}\right)\right] / \max \left(\theta_{\mathrm{j}}\right)-\min \left(\theta_{\mathrm{j}}\right)
\end{gathered}
$$

Then, the phase diagrams of each joint were derived from $\theta$ jnorm and wjnorm at each time point. According to the phase diagram, PA of the joint at each time point was determined to be the four-quadrant arctangent angle between the normalized angular 
velocity and the joint angle (PA $\varphi 1)$. Finally, the difference of the PA between the joints was calculated by the RPA, and thus the interjoint coordination was calculated.

\subsection{Statistical Analysis}

All statistical analysis was calculated using Matlab software (version R2019a; MathWorks, Inc., Natick, MA, USA), and analyses of variance were executed to compare parameters between DJH30, DJH40 and DJH50. Variables were analysed using a one-way ANOVA repeated measure for three drop heights. For the ANOVA results, sphericity was tested using Mauchly's test of sphericity. The Greenhouse-Geisser correction factor was used when aspheric results were present. If significant difference did exist, the least-significant difference's (LSD) pairwise post-hoc test was used to assess differences between individual heights. The significance level was set as $\alpha=0.05$ throughout the study. The effect size (ES) was used to determine whether a difference is actually a correlated difference. The variation differences of the three increasing drop heights was determined by the modified Cohen scale, the range of trivial difference is $<0.2$, the range of small difference is $0.2-0.6$, the range of moderate difference is $0.6-1.2$, and the range of large difference is 1.2-2.0 [33].

\section{Results}

Figure 1 presents the mean deviations of each dependent MARP variable. For the right and left hip-knee joints, there was shown to be more out-of-phase interjoint coordination (higher MARP) over the full landing phase in DJH30 and DJH40. The MARP of right and left hip-knee joints decreased significantly overall (all $p<0.050$ ) on the three increasing drop heights, with the post-hoc results showing differences between DJH30, DJH40, and DJH50. The post-hoc comparisons showed that the right hip-knee MARP were 33.76\%, $45.93 \%, 44.12 \%, 35.88 \%$, and $39.45 \%$ (all $p<0.018$; ES varying from 0.585 to 0.859 ) lower of DJH50 than DJH30, 37.21\%, 41.77\%, 26.24\%, 28.00\%, and 37.04\% (all $p<0.043$; ES varying from 0.488 to 1.036) lower of DJH50 than DJH40 at DJ1, DJs50, DJs100, DJs150 and DJs200, respectively (Figure 1A). The post-hoc comparisons showed that left hip-knee MARP were $31.87 \%, 49.82 \%, 41.91 \%, 44.78 \%$ and $42.83 \%$ (all $p<0.010$; ES varying from 0.652 to 1.163 ) lower of DJH50 than DJH30, 34.63\%, 45.44\%, 30.91\%, 44.97\% and 38.06\% (all $p<0.025$; ES varying from 0.547 to 1.108 ) lower of DJH50 than DJH40 at DJ1, DJs50, DJs100, DJs150 and DJs200, respectively (Figure 1B). There were no significant differences in right and left hip-ankle or knee-ankle of MARP between DJH30, DJH40 and DJH50 (Figure 1C-F). To sum up, the MARP of hip-knee varies with drop heights.

Figure 2 presents the mean deviations of each dependent DP variable. The right and left hip-knee joint showed more variability in interjoint coordination (DP) over the full landing phase in DJH30 and DJH40. The DP of right and left hip-knee jointd decreased significantly, overall (all $p<0.050$ ), on the three increasing drop heights, with the post-hoc results showing differences between DJH30, DJH40 and DJH50. The post-hoc comparisons showed that the right hip-knee DP were 29.13\%, 41.69\%, 48.32\%, 34.74\% and 29.83\% (all $p<0.044$; ES varying from 0.484 to 0.793 ) lower of DJH50 than DJH30, 41.74\%, 40.93\%, $34.18 \%, 23.88 \%$ and $36.75 \%$ (all $p<0.042$; ES varying from 0.491 to 0.803 ) lower of DJH50 than DJH40 at DJ1, DJs50, DJs100, DJs150 and DJs200, respectively (Figure 2A). The posthoc comparisons showed that left hip-knee DP were 35.95\%, 49.17\%, 41.09\%, 36.91\% and $36.43 \%$ (all $p<0.012$; ES varying from 0.625 to 1.046) lower of DJH50 than DJH30, 39.30\%, $39.02 \%, 36.50 \%, 26.45 \%$ and $26.70 \%$ (all $p<0.036$; ES varying from 0.501 to 0.665 ) lower of DJH50 than DJH40 at DJ1, DJs50, DJs100, DJs150 and DJs200, respectively (Figure 2B). There were no significant differences in right and left hip-ankle, knee-ankle of DP between DJH30, DJH40 and DJH50 (Figure 2C-F). In general, the difference of drop heights leads to the change of hip-knee DP.

Figure 3 presents the mean deviations of each dependent asymmetry variable. The side-to-side asymmetry of GCT increased significantly, overall (all $p<0.050$ ), on the three increasing drop heights, with the post-hoc results showing differences between DJH30, DJH40 and DJH50. The post-hoc comparisons showed that side-to-side asymmetry of 
GCT were 1.70-, 1.70-, 1.67-, 1.56- and 1.60-fold (all $p<0.043$; ES varying from 0.488 to 1.065) higher of DJH50 than DJH30, 1.89-, 1.55-, 1.88-, 1.75- and 1.78-fold (all $p<0.030$; ES varying from 0.528 to 1.042) higher of DJH50 than DJH40 at DJ1, DJs50, DJs100, DJs150 and DJs200, respectively (Figure 3A). The side-to-side asymmetry of peak I-vGRFpeak increased significantly, overall (all $p<0.050$ ), on the three increasing drop heights, with the post-hoc results showing differences between DJH30, DJH40 and DJH50. Our results show that side-to-side asymmetry of I-vGRFpeak were 1.54-, 3.05-, 1.92-, 1.57- and 1.74-fold higher (all $p<0.026$; ES varying from 0.546 to 1.059) during DJH40 than DJH30; and 2.10-, 2.76-, 1.93-, 1.89- and 2.28-fold higher (all $p<0.016$; ES varying from 0.598 to 0.847 ) during DJH50 than DJH30 at DJ1, DJs50, DJs100, DJs150 and DJs200, respectively (Figure 3B).
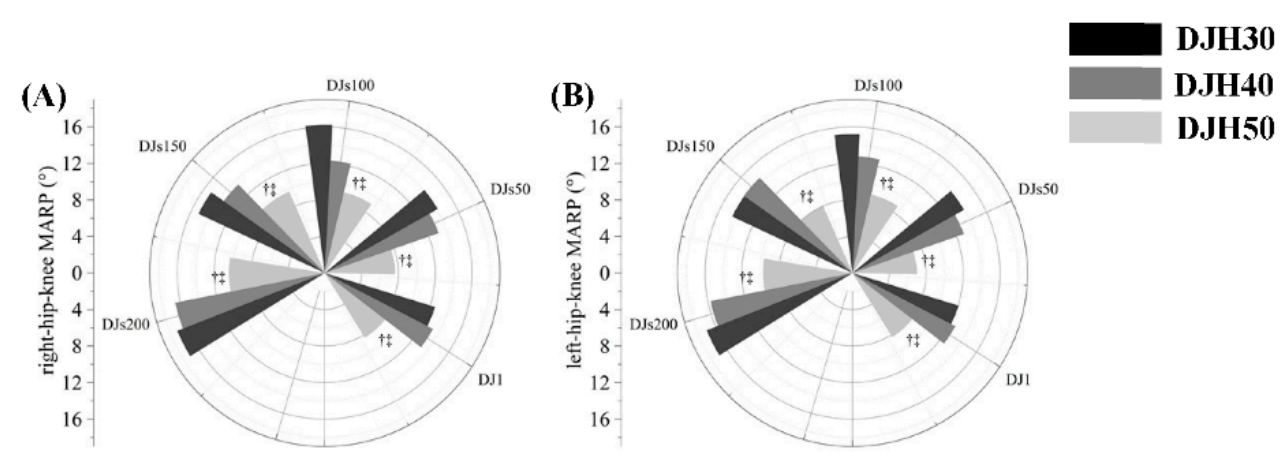

DJH30
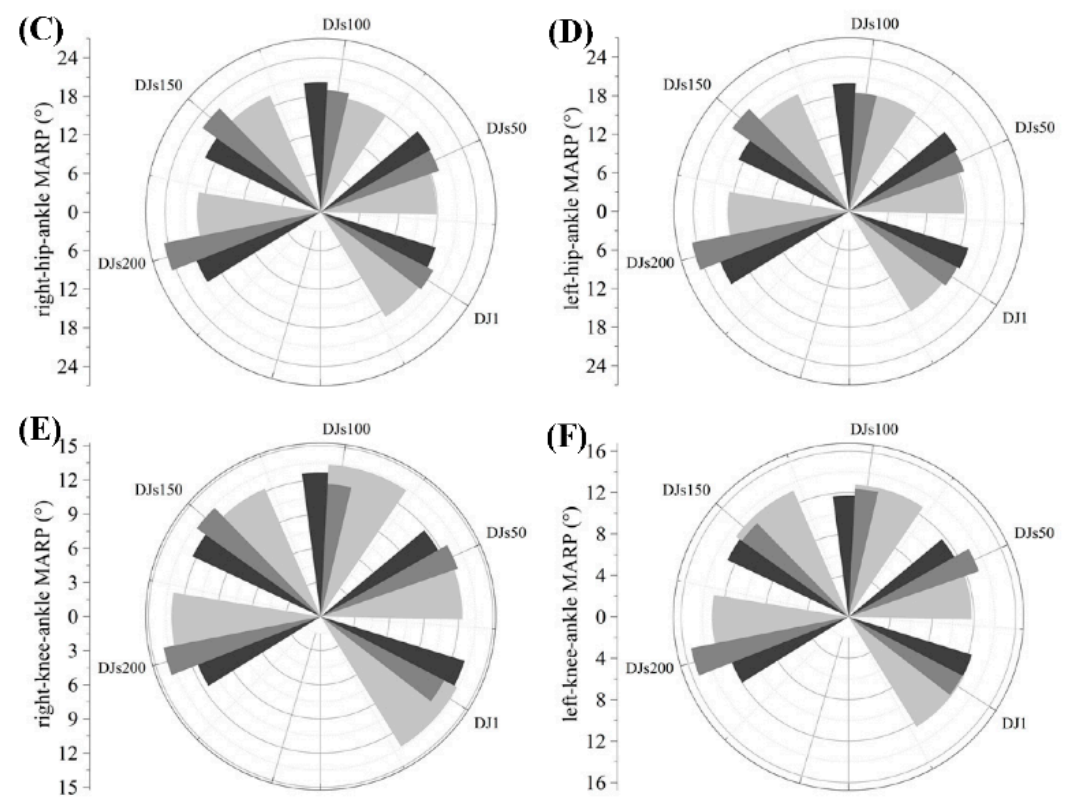

Figure 1. MARP = mean absolute relative phase. (A,C,E) represent the right MARP of hip-knee, hip-ankle and knee-ankle respectively, (B,D,F) represent the right MARP of hip-knee, hip-ankle and knee-ankle respectively during drop-jumps from three heights at DJ1, DJs50, DJs100, DJs150, and DJs200. Asterisk + indicates that a significant difference with DJH30; $\ddagger$ indicates that a significant difference with DJH40. $p$ values $<0.05$ were considered to significantly differ. 

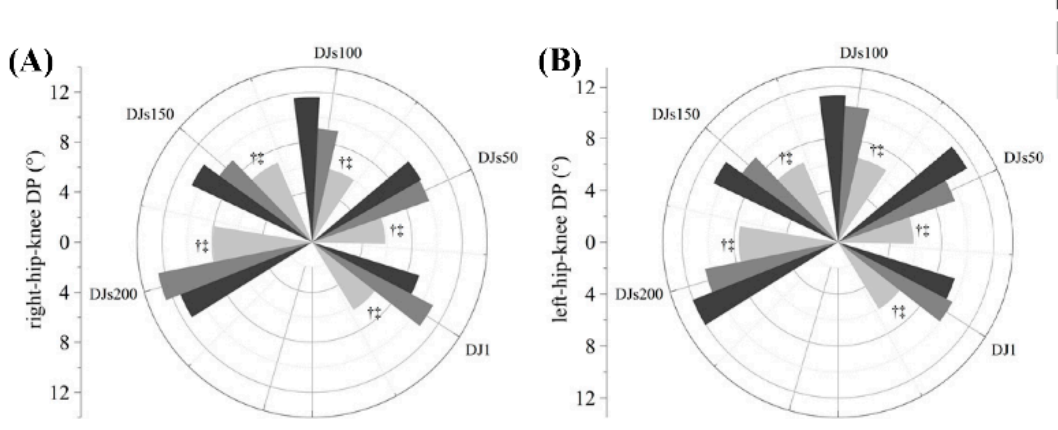

D.JH30

D.JH40

D.JH50
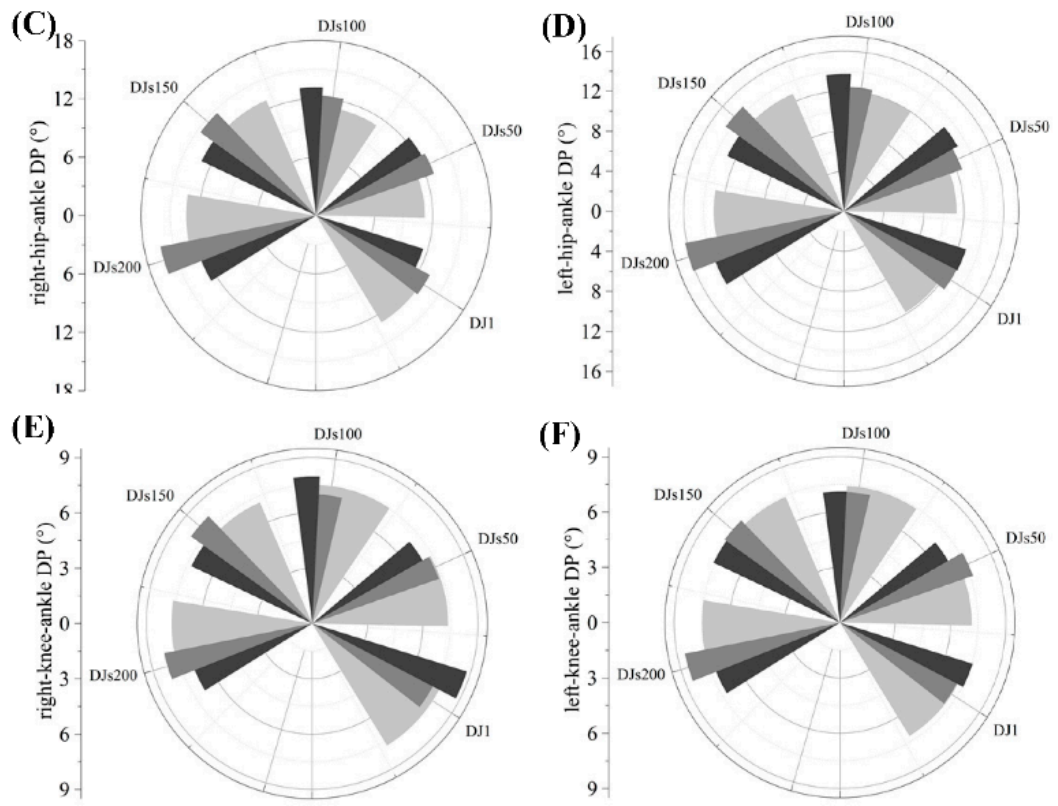

Figure 2. DP = deviation phase. (A,C,E) represent the right DP of hip-knee, hip-ankle and knee-ankle respectively, $(\mathbf{B}, \mathbf{D}, \mathbf{F})$ represent the right DP of hip-knee, hip-ankle and knee-ankle respectively during drop-jumps from three heights at DJ1, DJs50, DJs100, DJs150 and DJs200. Asterisk + indicates that a significant difference with DJH30; $\ddagger$ indicates that a significant difference with DJH40. $P$ values $<0.05$ were considered to significantly differ.

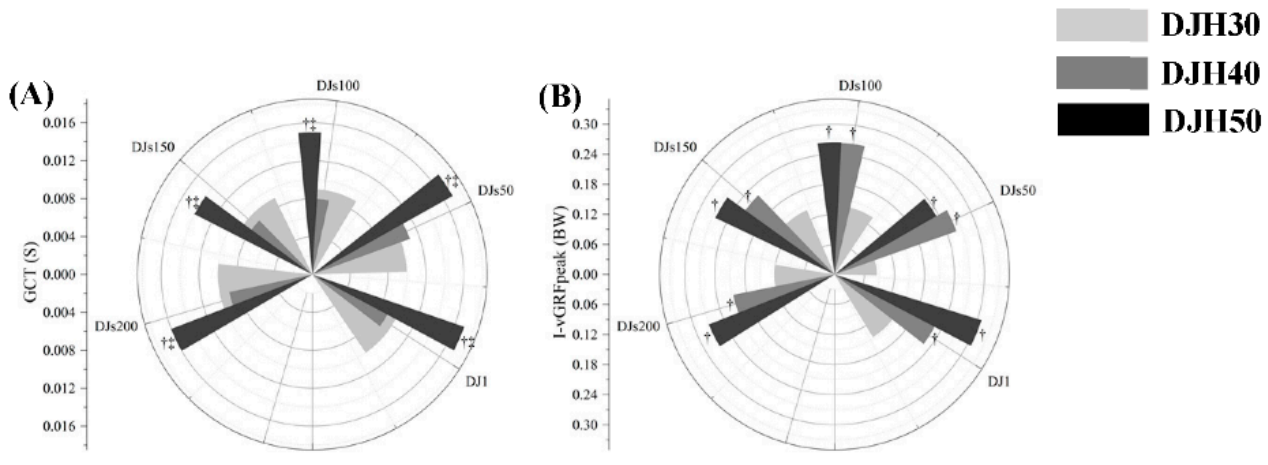

Figure 3. GCT = ground contact time; I-vGRFpeak = peak impact force during the landing phase. $(\mathbf{A}, \mathbf{B})$ represent the side-to-side asymmetry of GCT and I-vGRFpeak respectively during drop-jumps from three heights at DJ1, DJs50, DJs100, DJs150 and DJs200. Asterisk + indicates that a significant difference with DJH30; $\ddagger$ indicates that a significant difference with DJH40. $p$ values $<0.05$ were considered to significantly differ. 


\section{Discussion}

This study presented a comparative investigation between MARP and DPs in the lower extremity for different drop heights and training volumes while performing a DJ task. Consistent with the hypothesis, the results show higher DP and MARP of hip-knee joints and higher side-to-side asymmetry of GCT at DJH30 and DJH40. The side-to-side asymmetry of I-vGRFpeak was higher at DJH50.

The study found that MARPs of right and left hip-knee joints, at DJH30 and DJH40, were greater than DJH50 within DJs200. Changes in initial posture may affect muscletendon unit lengths and generate different forces, and joint moments may affect MARP [34]. The lower the angle of the knee during DJ-landing from higher drop heights results in a different initial position during takeoff [35]. Thus, changes in initial position during landing from different drop heights may alter muscle-tendon length and cause the difference in MARP. However, MARP was not changed in the hip-ankle and knee-ankle joints, at different drop heights. The important angular moments caused by the "prioritizing"of a hip-knee coordination-motion-control strategy contributes to improving athletic performance [34]. In addition, a lower MARP results in difficulty in movement control and error correction during joint movement [36]. Therefore, the drop heights of 30 and $40 \mathrm{~cm}$ showed better interjoint coordination of hip-knee joints within DJs200.

The higher DP of the right and left hip-knee joints within DJs200, in DJH30 and DJH40, compared with DJH50, indicates that drop heights may affect DP and there is a greater coordination instability for the hip-knee joint at lower drop heights. Prior research has found that higher DPs may be a functional feature of the neuromusculoskeletal system that better redistributes anatomical load and reduces the risk of injury by reducing repetitive patterns [12,37]. Accordingly, the higher DP for hip-knee joints in DJH30 and DJH40 may better redistribute lower-extremity joint load effectively, and thereby reduce lowerextremity injury. Morever, a lower DP is consistent with protective behavior in terms of physical performance [38]. The DP variation caused by larger ground impact forces during landing may result in less strict control over joint couplings [39]. Therefore, when landing during DJH50, a lower DP for hip-knee joints is not conductive to joint control, but may be a protective strategy for athletes to cope with high-impact forces. However, in this study, the DP of hip-ankle and knee-ankle joints di not differ by drop height. It may be that the three drop heights and training volumes used were not sufficiently threatening to trigger protective reinforcement motor strategies for the hip-ankle and knee-ankle joints during landing. Thus, a greater DP of hip-knee joints during DJH30 and DJH40 may reduce injury in the lower extremities, and a DP in DJH50 may be due to a protective strategy for coping with the larger ground impact in DJs200.

The results of this study show that the side-to-side asymmetry of GCT increases with drop height. Previous studies have found that differences in muscle strength, flexibility, and dynamic control of the lower extremities lead to increased side-to-side asymmetry of GCT, which affects the benefit of training $[4,40,41]$. Thus, as side-to-side asymmetry of GCT increases with drop heights—especially at DJH50 — may affect training efficacy. In addition, limb preference may play a role in some of the kinetic differences between limbs during DJ [42]. The higher I-vGRFpeak in one limb seems to indicate that the limb bore more weight on landing indicates functional dominance for that limb compared with the other [21]. Overuse of the dominant foot can put more pressure on the corresponding knee joint, leaving the nondominant leg unable to absorb vibration effectively [43]. Athletes who regularly jump during training may gradually overload the functional limbs, leading to impacts such as chronic injury [21]. Therefore, the higher side-to-side asymmetry of I-vGRFpeak may increase injury risk during DJH50. Accordingly, the smaller side-to-side asymmetry of GCT and I-vGRFpeak during DJH30 are beneficial to reduce the risk of training injury and improve the training effect. 


\section{Conclusions}

We concluded that interjoint coordination patterns, variability and side-to-side asymmetry were affected by drop height in drop jumping. MARP and DP were higher when jumps were performed within both DJH30 and DJH40. Also, changes, verified by CRP, indicated that different drop heights affected intersegmental coordination. This suggests that when subjects assume different drop heights in a drop-jump it may interfere with their motor patterns. Different drop heights and training volumes during DJ training will affect side-to-side asymmetry and interjoint coordination during landing. Therefore, coaches should take care to choose the appropriate drop height and training volume when DJ training their athletes.

\section{Limitation}

There were limitations to this study; we did not collect data on women. Future work will considered gender as a mediating factor in the analysis of different drop height and training volume on the biomechanics of the lower extremity.

Author Contributions: Conceptualization, I.-L.W.; Writing-original draft preparation, K.-K.Z., I.L.W. and Y.-M.C.; software, Y.-G.L.; formal analysis, Y.S. and S.Y.; Writing-review \& editing I.-L.W., K.-K.Z. All authors have read and agreed to the published version of the manuscript.

Funding: This work was supported by Research on Scientific Research Project of Hubei Provincial Department of Education of Effects of Customized Functional In-soles on the Lower-Limb Biomechanics of Short Track Skaters [grant number D20202502].

Institutional Review Board Statement: The study was conducted in accordance with the guidelines of the Declaration of Helsinki and approved by the Institutional Review Board (JLSU-IRB2020004, 2020-09-01).

Informed Consent Statement: The study was approved by the regional ethics committee, and all subjects signed informed consent forms.

Data Availability Statement: The datasets used and analyzed in the current study are included in this article.

Conflicts of Interest: The authors declare no conflict of interest.

\section{References}

1. Matavulj, D.; Kukolj, M.; Ugarkovic, D.; Tihanyi, J. Effects of pylometric training on jumping performance in junior basketball players. J. Sports Med. Phys. Fitness. 2001, 41, 159-164. [PubMed]

2. Holcomb, W.R.; Lander, J.E.; Rutland, R.M.; Wilson, G.D. The effectiveness of a modified plyometric program on power and the vertical jump. J. Strength Cond. Res. 1996, 10, 89-92.

3. Wilson, G.J.; Newton, R.U.; Murphy, A.J.; Humphries, B.J. The optimal training load for the development of dynamic athletic performance. Med. Sci. Sports Exerc. 1993, 25, 1279-1286. [CrossRef] [PubMed]

4. Ball, N.B.; Stock, C.G.; Scurr, J.C. Bilateral contact ground reaction forces and contact times during plyometric drop jumping. J. Strength Cond. Res. 2010, 24, 2762-2769. [CrossRef]

5. Mrdakovic, V.; Ilic, D.B.; Jankovic, N.; Rajkovic, Z.; Stefanovic, D. Pre-activity modulation of lower extremity muscles within different types and heights of deep jump. J. Sports Sci. Med. 2008, 7, 269.

6. Ruan, M.; Li, L. Influence of a horizontal approach on the mechanical output during drop jumps. Res. Q. Exerc. Sport 2008, 79, 1-9. [CrossRef]

7. Peng, H.-T. Changes in biomechanical properties during drop jumps of incremental height. J. Strength Cond. Res. 2011, 25, 2510-2518. [CrossRef] [PubMed]

8. Peng, H.-T.; Kernozek, T.W.; Song, C.-Y. Quadricep and hamstring activation during drop jumps with changes in drop height. Phys. Ther. Sport 2011, 12, 127-132. [CrossRef] [PubMed]

9. Wallace, B.J.; Kernozek, T.W.; White, J.M.; Kline, D.E.; Wright, G.A.; Peng, H.-T.; Huang, C.-F. Quantification of vertical ground reaction forces of popular bilateral plyometric exercises. J. Strength Cond. Res. 2010, 24, 207-212. [CrossRef]

10. Malinzak, R.A.; Colby, S.M.; Kirkendall, D.T.; Yu, B.; Garrett, W.E. A comparison of knee joint motion patterns between men and women in selected athletic tasks. Clin. Biomech. 2001, 16, 438-445. [CrossRef]

11. Chockley, C. Ground reaction force comparison between jumps landing on the full foot and jumps landing en pointe in ballet dancers. J. Dance Med. Sci. 2008, 12, 5-8. 
12. Hamill, J.; van Emmerik, R.E.; Heiderscheit, B.C.; Li, L. A dynamical systems approach to lower extremity running injuries. Clin. Biomech. 1999, 14, 297-308. [CrossRef]

13. Schoner, G.; Kelso, J. Dynamic pattern generation in behavioral and neural systems. Science 1988, 239, 1513-1520. [CrossRef]

14. Scholz, J.P. Dynamic pattern theory—some implications for therapeutics. Phys. Ther. 1990, 70, 827-843. [CrossRef]

15. Williams, G.N.; Chmielewski, T.; Rudolph, K.S.; Buchanan, T.S.; Snyder-Mackler, L. Dynamic knee stability: Current theory and implications for clinicians and scientists. J. Orthop. Sports Phys. Ther. 2001, 31, 546-566. [CrossRef]

16. Knihs, D.A.; Zimmermann, H.B.; Dal Pupo, J. Acute and Delayed Effects of Fatigue on Ground Reaction Force, Lower Limb Stiffness and Coordination Asymmetries During a Landing Task. J. Hum. Kinet. 2021, 76, 191. [CrossRef] [PubMed]

17. Aerts, I.; Cumps, E.; Verhagen, E.; Verschueren, J.; Meeusen, R. A systematic review of different jump-landing variables in relation to injuries. J. Sports Med. Phys. Fitness. 2013, 53, 509-519. [PubMed]

18. Bartlett, R.; Wheat, J.; Robins, M. Is movement variability important for sports biomechanists? Sports Biomech. 2007, 6, 224-243. [CrossRef]

19. Pupo, J.D.; Dias, J.A.; Gheller, R.G.; Detanico, D.; Santos, S.G.D. Stiffness, intralimb coordination, and joint modulation during a continuous vertical jump test. Sports Biomech. 2013, 12, 259-271. [CrossRef]

20. Sinsurin, K.; Srisangboriboon, S.; Vachalathiti, R. Side-to-side differences in lower extremity biomechanics during multi-directional jump landing in volleyball athletes. Eur. J. Sport Sci. 2017, 17, 699-709. [CrossRef] [PubMed]

21. Lim, Y.Y.; Sterzing, T.; Teo, C.J.; Alonzo, R.; Pan, J.W.; Teng, P.S.; Kong, P.W. Between-limb asymmetry in kinetic and temporal characteristics during bilateral plyometric drop jumps from different heights. J. Sports Sci. 2020, 38, 1605-1614. [CrossRef] [PubMed]

22. Ball, N.B.; Scurr, J.C. Bilateral neuromuscular and force differences during a plyometric task. J. Strength Cond. Res. 2009, 23, 1433-1441. [CrossRef] [PubMed]

23. Rannama, I.; Port, K.; Bazanov, B.; Pedak, K. Sprint cycling performance and asymmetry. J. Hum. Sport Exerc. 2015, 10, S248-S258. [CrossRef]

24. Bishop, C.; Pereira, L.A.; Reis, V.P.; Read, P.; Turner, A.N.; Loturco, I. Comparing the magnitude and direction of asymmetry during the squat, countermovement and drop jump tests in elite youth female soccer players. J. Sports Sci. 2020, 38, 1296-1303. [CrossRef]

25. Gu, C.-Y.; Li, X.-R.; Lai, C.-T.; Gao, J.-J.; Wang, I.-L.; Wang, L.-I. Sex Disparity in Bilateral Asymmetry of Impact Forces during Height-Adjusted Drop Jumps. Int. J. Environ. Res. Public Health. 2021, 18, 5953. [CrossRef]

26. Wang, I.-L.; Chen, Y.-M.; Zhang, K.-K.; Li, Y.-G.; Su, Y.; Wu, C.; Ho, C.-S. Influences of Different Drop Height Training on Lower Extremity Kinematics and Stiffness during Repetitive Drop Jump. Appl. Bionics. Biomech. 2021, 2021, 5551199. [CrossRef]

27. Wang, I.-L.; Hsiao, C.-Y.; Shen, J.; Wang, Y.; Huang, C.-C.; Chen, Y.-M. The effects of Jilin sika Deer's (Cervus dybowski) tendon liquid supplementation on endurance drop jumps performance, biochemistry profile of free boxing players. J. Ethnopharmacol. 2019, 245, 112119. [CrossRef]

28. Wang, I.-L.; Wang, S.-Y.; Wang, L.-I. Sex differences in lower extremity stiffness and kinematics alterations during double-legged drop landings with changes in drop height. Sports Biomech. 2015, 14, 404-412. [CrossRef]

29. Wang, I.-L.; Hsiao, C.-Y.; Li, Y.-H.; Meng, F.-B.; Huang, C.-C.; Chen, Y.-M. Nanobubbles water curcumin extract reduces injury risks on drop jumps in women: A pilot study. Evid. Based Complementary Altern. Med. 2019, 2019, 8647587. [CrossRef]

30. Ippersiel, P.; Robbins, S.; Preuss, R. Movement variability in adults with low back pain during sit-to-stand-to-sit. Clin. Biomech. 2018, 58, 90-95. [CrossRef]

31. Scholes, C.J.; McDonald, M.D.; Parker, A.W. Single-subject analysis reveals variation in knee mechanics during step landing. J. Biomech. 2012, 45, 2074-2078. [CrossRef]

32. Frayne, D.H.; Zettel, J.L.; Beach, T.A.; Brown, S.H. The Influence of Countermovements on Inter-Segmental Coordination and Mechanical Energy Transfer during Vertical Jumping. J. Mot. Behav. 2020, 53, 1-13. [CrossRef]

33. Hopkins, W.; Marshall, S.; Batterham, A.; Hanin, J. Progressive statistics for studies in sports medicine and exercise science. Med. Sci. Sports Exerc. 2009, 41, 3. [CrossRef]

34. Gheller, R.G.; Dal Pupo, J.; Ache-Dias, J.; Detanico, D.; Padulo, J.; dos Santos, S.G. Effect of different knee starting angles on intersegmental coordination and performance in vertical jumps. Hum. Mov. Sci. 2015, 42, 71-80. [CrossRef]

35. Fagenbaum, R.; Darling, W.G. Jump landing strategies in male and female college athletes and the implications of such strategies for anterior cruciate ligament injury. Am. J. Sports Med. 2003, 31, 233-240. [CrossRef] [PubMed]

36. Latash, M.L.; Scholz, J.P.; Schöner, G. Motor control strategies revealed in the structure of motor variability. Exerc. Sport Sci. Rev. 2002, 30, 26-31. [CrossRef]

37. Preatoni, E.; Hamill, J.; Harrison, A.J.; Hayes, K.; Van Emmerik, R.E.; Wilson, C.; Rodano, R. Movement variability and skills monitoring in sports. Sports Biomech. 2013, 12, 69-92. [CrossRef]

38. Moseley, G.L.; Hodges, P.W. Reduced variability of postural strategy prevents normalization of motor changes induced by back pain: A risk factor for chronic trouble? Behav. Neurosci. 2006, 120, 474. [CrossRef] [PubMed]

39. Cazzola, D.; Pavei, G.; Preatoni, E. Can coordination variability identify performance factors and skill level in competitive sport? The case of race walking. J. Sport Health Sci. 2016, 5, 35-43. [CrossRef] 
40. Hewett, T.E.; Myer, G.D.; Ford, K.R.; Heidt, R.S., Jr.; Colosimo, A.J.; McLean, S.G.; Van den Bogert, A.J.; Paterno, M.V.; Succop, P. Biomechanical measures of neuromuscular control and valgus loading of the knee predict anterior cruciate ligament injury risk in female athletes: A prospective study. Am. J. Sports Med. 2005, 33, 492-501. [CrossRef] [PubMed]

41. Ford, K.R.; Myer, G.D.; Hewett, T.E. Valgus knee motion during landing in high school female and male basketball players. Med. Sci. Sports Exerc. 2003, 35, 1745-1750. [CrossRef] [PubMed]

42. Britto, M.A.d.; Franco, P.S.; Pappas, E.; Carpes, F.P. Kinetic asymmetries between forward and drop jump landing tasks. Rev. Bras. Cineantropom. Desempenho Hum. 2015, 17, 661-671.

43. Hewett, T.E.; Ford, K.R.; Hoogenboom, B.J.; Myer, G.D. Understanding and preventing acl injuries: Current biomechanical and epidemiologic considerations-update 2010. N. Am. J. Sports Phys. Ther. 2010, 5, 234. [PubMed] 